\title{
Signal Efficiency in Gradient Index Lens Based Two Photon Microscopy
}

\author{
Axel Wingert ${ }^{1}$, Henning Seim ${ }^{1}$, Sebastian Schürmann ${ }^{1 *}$, Rainer H. A. Fink ${ }^{1}$, Martin Vogel ${ }^{2}$ \\ ${ }^{1}$ Medical Biophysics, Institute of Physiology and Pathophysiology, University of Heidelberg, Heidelberg, Germany \\ ${ }^{2}$ Max Planck Institute of Biophysics, Frankfurt, Germany \\ Email: axelwingert@hotmail.com,m@rtin-vogel.de
}

Received December 24, 2012; revised January 25, 2013; accepted February 10, 2013

\begin{abstract}
Gradient index (GRIN) lenses are often used as an optical relay to a sample at a location that is not accessible for a standard microscope. This capability is turning them into an important enabling technology that extends many optical imaging modalities like harmonic laser scanned imaging with micro endoscopic in vivo capabilities as needed in research and diagnostics. These micro endoscopic imaging variants however rely on the light scattering capability of the underlying tissue. Further complications arise from an increased number of optical interfaces and the overall optical performance of a GRIN rod. We have therefore performed a quantitative comparison of the back-scattered second harmonic generation (SHG) signal intensity generated in skin and low-scattering muscle tissue, both obtained with a standard two photon laser scanning microscope (LSM) and a GRIN lens based LSM. We report that the GRIN lens based system sees approximately $1 / 4$ of the net two photon signal detected by the standard LSM. We expect that this value can be generalized to other LSM techniques enhanced by GRIN technology and encourage its use in experimental situations with standard LSM signal to noise ratios of four or higher.
\end{abstract}

Keywords: Second Harmonic Generation; Gradient Index Lens; Multi Photon Microscopy; Multi Harmonic Microscopy; Micro-Endoscopy

\section{Introduction}

Since more than a century, optical microscopy is one of the major tools to analyze structure and function in biomedical applications on a micrometer scale. Yet, the relative opaqueness of the tissue in the visible and near-infrared ranges of wavelengths and the relative size of the objective lenses make it difficult to deploy optical microscopy in layers deep inside the sample.

Gradient index (GRIN) lenses are of major interest in this setting as they enable micro-endoscopic applications [1-6]. As an example, an important micro-endoscopic application has been developed recently to image the contraction process of human muscle fibers in vivo [7] which led to new insights into the contractile dynamics of sarcomeres. In the field of muscle physiology alone, GRIN lens technique has many possible applications, e.g., research on continuously progressing muscular diseases like Duchenne muscle dystrophy [8,9], but also diagnosis and rehabilitation control of acute ruptures of muscle fibers or entire muscles which still rely on diagnostic tools like CT, NMR or sonography that do not provide image resolution at the sarcomere level. The same is true

"Current address: Medical Biotechnology, Friedrich-Alexander-University, Erlangen, Germany. for the diagnostics of the healing progress after cross bar ruptures or the classification of various possible achillodynia syndroms which currently relies on the same tools. In general, it is very likely that GRIN lenses will find use as an enabling technology for intra-tissue and microendoscopic imaging in all fields of biology and biomedicine.

GRIN lenses are typically used as an optical relay where an intermediate object plane of an objective lens is re-imaged through the GRIN rod into the tissue. This scheme will of course affect the signal efficiency of the apparatus, i.e. the detected signal versus light power input. To know this level of efficiency in comparison to well-characterized standard microscopes is an important datum for the development of micro endoscopic devices based on GRIN lens technology and to make use of the huge amount of knowledge and quantitative image data available from decades of research.

In biomedical tissue imaging, laser-scanned two-photon excitated fluorescence microscopy (TPEF) [10] is often used due to its larger penetration depth, optical sectioning capability and lower background compared with other bright field or single photon laser scanning imaging methods. Yet, the application of imaging modalities 
to an in vivo setting with thick and therefore almost opaque samples behind the imaging apparatus often has to exploit the sample property of light back-scattering $[11,12]$ and to guide both excitation and emission light paths through the same nose-piece optics $[13,14]$. The use of a mirror in a position below the image plane has been proposed to increase the detected signal intensity [15], but it remains open whether such a configuration can be used in all experimental or even in in vivo diagnostic settings. We have therefore set out to quantify the signal efficiency and relative level of signal $R$ comparing both a standard SHG/two-photon and a GRIN lens based two photon laser scanning system in the backscattering signal detection mode. We used a GRIN system known to us as the only off the shelf product especially designed for medical applications (GRIN-GT-MO-080018-810, Grintech $\mathrm{GmbH}$, Jena, Germany) and have investigated its performance comparing the net signal obtained in laser scanning SHG imaging on both systems.

Within the last decade, Second Harmonic Generation (SHG) microscopy has emerged as a tool for tissue imaging, often deploying label-free detection of filamentous protein structures like collagen or myosin [16-19] whose ubiquity renders them to very important and useful image mapping agents in biomedicine. A technical cousin to TPEF, the SHG modality is available on every two-photon microscope, provided that appropriate optical filters are installed, and shows the same advantages like increased penetration depth, optical sectioning capability and background suppression.

Second Harmonic Generation (SHG) signals allow high resolution microscopy in skin or muscle tissue [20-22]. Since the signal is created intrinsically by the collagen and myosin filaments extrinsic staining is unnecessary. In addition, SHG signals show no bleaching, so that they can be used well for quantitative signal measurements and comparisons. On the other hand, SHG signals have two unusual properties, a significant dependence on laser polarization and sample-dependent signal emission asymmetry.

Keeping the sample orientation aligned with the laser polarization is a straight forward control to the polarization dependence of the SHG signal. Due to its nature as a coherent process, however, the angular radiation pattern of the SHG light will — to our advantage - highly depend on the distribution of second harmonic scatterers in the laser focus. For a "volume-like" distribution as in myosin, the- ory predicts that the SHG signal is generated mostly off- axis, yet dominantly in the forward direction; for more point-like distributions like in collagen, there is more backward generated signal $[23,24]$.

Accordingly, in skeletal muscles a large percentage of the signal detected in the backward direction is actually generated in forward direction but scattered back by un- derlying layers of tissue $[25,26]$ leading to a rather weak yet reasonably detectable signal. Thus, using SHG signals from thick glycerinated, i.e. "optically cleared" and therefore low-scattering muscle fibers [26-28] allows us to establish a less favoring experimental test case for backscattered signal imaging with GRIN lenses.

Since connective tissue and collagen also produce SHG signal directly in the backward direction and also with higher intensity than muscle fibers [25] imaging thin samples of skin tissue set a second, more favoring test case for a GRIN lens based endoscopic system.

\section{Materials and Methods}

\subsection{Sample Preparation}

We used skin and muscle preparations from C57BL6 mice that were handled according to the regulations set up by the local animal care committee. Skin samples were obtained by embedding skin tissue in Tissue Tek (Sakura Finetek, Staufen, Germany) and freezing the tissue block in liquid nitrogen. Thin slices a few micrometers in thickness were obtained with a cryo-microtome and mounted on a cover slip.

To prevent contraction and to facilitate the storage of functionally intact samples, freshly prepared EDL (Extensor Digitorum Longus) muscle was fixed at both ends on a rod and subsequently glycerinated and "optically cleared" in "skinning solution" (50\% glycerol with 10 mM DTT, 50\% solution containing $40 \mathrm{mM}$ HEPES, 20 mM EGTA, $8.8 \mathrm{mM} \mathrm{MgOH,} 8$ mM ATP, $10 \mathrm{mM} \mathrm{CP}$, pH 7.0) for more than 5 hours in a cold room environment, with eventual subsequent storage at $-20^{\circ} \mathrm{C}$ for later use. Fiber bundles from stored EDL were then prepared in rigor solution (10 mM HEPES, $2 \mathrm{mM}$ EGTA, $10 \mathrm{mM}$ $\mathrm{MgCl}, 140 \mathrm{mM}$ potassium glutamate, $\mathrm{pH}$ 7.0) and their ends fixed on a cover slip with sticky tape. The coverslips were set into a custom-built chamber that allowed to fill the space over the sample with rigor solution up to 25 $\mathrm{mm}$ in height in order to suppress light reflections.

\subsection{SHG Imaging}

The chamber was mounted on an inverted microscope (DM IRBE, Leica Microsystems, Mannheim, Germany). SHG recordings were carried out as reported before with a picosecond-pulsed ( $\tau \approx 2$ ps) Ti:Sa laser (Tsunami, Spectra Physics, Irvine, CA) tuned to $880 \mathrm{~nm}$ serving as excitation source $[9,18,22,29]$. The excitation laser was focused onto the sample with a $63 \mathrm{x} / 1$.2NA water immersion lens (Leica HCX PL APO CS). The laser intensity was adjusted for optimal imaging and measured at the back aperture of the objective with typical values of 200 $300 \mathrm{~mW}$, see panel A of Figure 1.

Alternatively, the $63 \mathrm{x}$ lens has been replaced by a 
A

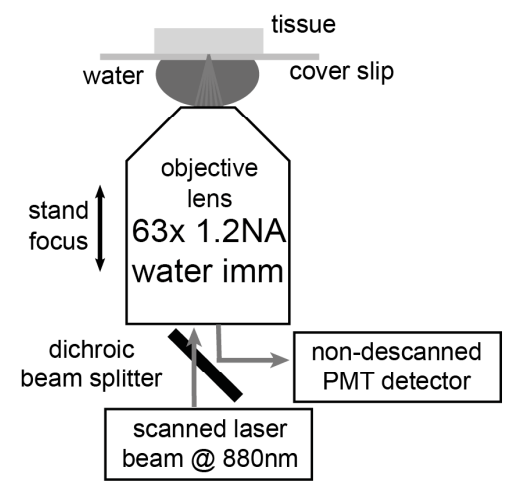

C

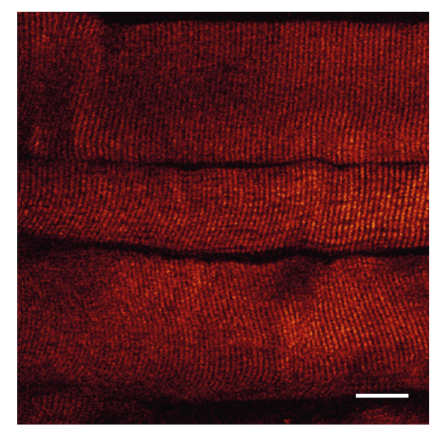

E

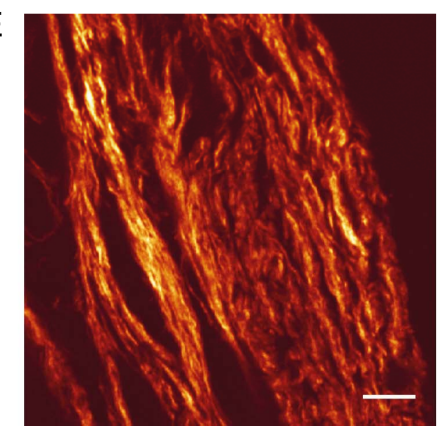

B

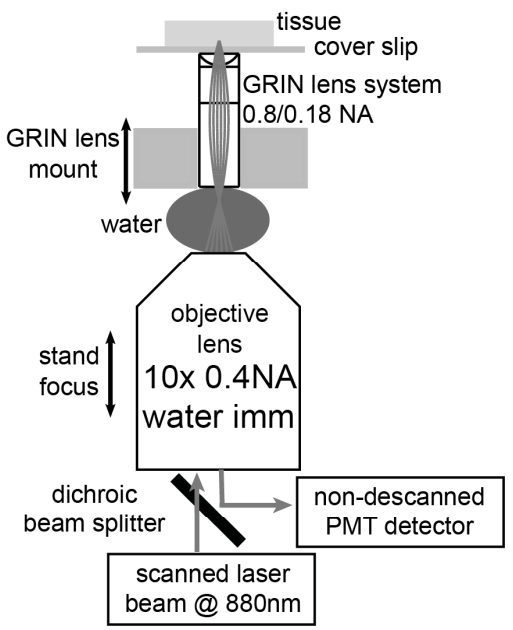

D

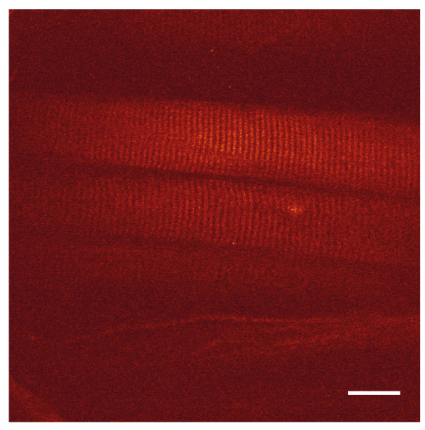

F

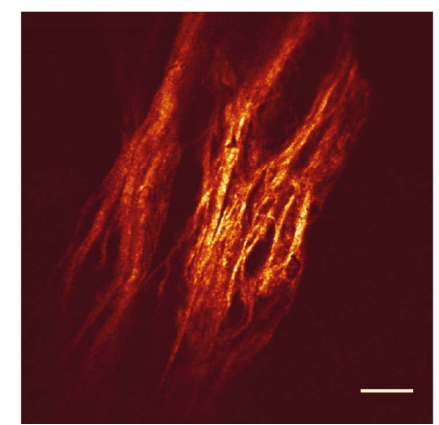

Figure 1. A: Objective lens system: The laser beam is focused to the sample with a 63x objective lens. B: GRIN lens system: The laser beam is focused and scanned in the back focal plane of the GRIN objective and the latter is relaying the laser focus into the sample. Objective and GRIN lens have independent means of $\mathrm{z}$ focusing; optical contact is made through water immersion. C: Back-scattered SHG image from glycerinated EDL muscle tissue recorded with the $63 \times 1.2$ NA microscope objective. D: Back-scattered SHG image from similar sample, but imaged through the GRIN lens system. E, F: Back-scattered SHG image from skin tissue recorded with the $63 \times 1.2$ NA microscope objective and through the GRIN lens system, respectively. Scale bar in panels C through F: $20 \mu \mathrm{m}$.

combination of a $10 \mathrm{x} / 0.4 \mathrm{NA}$ water immersion objective lens (Leica HC APO IMM) and the gradient index lens system (GRIN-GT-MO-080-018-810, Grintech), see panel B of Figure 1. Here, the laser beam is focused by the $10 \mathrm{x}$ lens in water immersion and scanned in the back focal plane of the GRIN lens, whereas the latter acts as an optical relay with a magnification of $\approx 4.8$ and working distance of $\approx 200 \mu \mathrm{m}$. On the sample and the lens side of the GRIN system, the central numerical apertures (NA) are 0.8 and 0.18 , respectively, and the lens has a field diameter of $\approx 200 \mu \mathrm{m}$ and $\approx 1 \mathrm{~mm}$, respectively.

With a beam diameter of approximately $5.5 \mathrm{~mm}$, we are underfilling the back aperture of both objective lenses which are approximately $14 \mathrm{~mm}$ and $8.5 \mathrm{~mm}$ in diameter for the 10x and the $63 \mathrm{x}$ lens, respectively, and we assume that there are no more substantial differences with respect to the excitation numerical aperture (see "Discussion").

The GRIN system is attached to the microscope with a custom built sample stage that enables us to move the 
GRIN objective in lateral directions; along the optical axis, the GRIN lens is fixed. Focusing is performed by $\mathrm{z}$ movement of both the microscope objective and the sample that is mounted on an adjustable stage. SHG images were acquired with approximately $300-350 \mathrm{~mW}$ laser power entering the back aperture plane of the $10 \mathrm{x}$ lens. As second harmonic generation has a quadratic dependence on the laser power, we were able to correct for data variances due to these varying laser power levels.

The long axes of the muscle fibers were positioned perpendicularly to the axis of the laser beam polarization. At this orientation the SHG polarization dependency curve has a local minimum $[18,22]$, so that minor alterations of the fiber orientation have a reduced effect on the signal intensity. Collagen fibers are less oriented in connected tissue but we have similarly tried to maintain a principal vertical alignment of the sample.

SHG light was collected with the same lens systems in backward direction, coupled out of the beam path directly beneath the objective lens with a dichroic mirror (700SP, Chroma Technology Corp., Bellows Falls, VT), directed towards the standard Leica epi non-descanned PMT unit which is located approximately $170 \mathrm{~mm}$ away from the lens back aperture position. Signal light was further filtered by a dichroic mirror (FF458-Di01, Semrock Inc., Rochester, NY) and a band pass filter (FF01-438/24, Semrock) and finally detected by a PMT (R9624, Hamamatsu, Hamamatsu City, Japan). The voltage dependence of the PMT amplification was calibrated at constant laser power with two photon excited signals from a chamber filled with fluorescein solution.

\section{Results}

\subsection{Theoretical Considerations}

The manufacturer does not release a detailed optical prescription of the GRIN lens system so that we had to resort to a number of assumptions based on the information on the specification sheet: The lens system consists of at least one GRIN rod element at the low NA (0.18) end, combined with a plan-convex glass lens at the high NA (0.8) end where the curved surface faces the GRIN rod.

For typical GRIN material, we assume an index of refraction of $\approx 1.6$, and $\approx 1.45$ to 1.55 for the glass lens. This range is deduced from the refractive index [30] of BK7 and fused silica glass at the two principal wavelengths of the study, $880 \mathrm{~nm}$ and $440 \mathrm{~nm}$. We further assume a numerical aperture of $\approx 0.5$ in the air-filled space between the GRIN rod and the glass elements, normal incidence on the curved glass surface and water immersion $(\mathrm{n}=$ 1.33 ) on both ends of the lens system.

Applying the well-known Fresnel formula for the reflection losses - averaged for arbitrary polarization-for a ray of light on the optical axis yields a theoretical esti- mate of 0.79 to 0.83 for the transmission of the GRIN system. Interestingly, extending this simple model to a whole fan of 75 rays $(\approx 1$ ray per degree on the high NA side) yields only a slight shift to 0.79 to 0.82 as all angles of incidence stay below $\approx 37$ degrees.

As the SHG signals shows a quadratic dependence on the incident laser power, the relative signal level $R_{\text {theo }}$ will scale with the cube on this transmission value. In addition, $R_{\text {theo }}$ will depend of the ratio of the numerical apertures. As we assume that the difference of the effective NA with respect to the excitation is negligible, $R_{\text {theo }}$ will only scale with the ratio of the signal collection capability of the lenses, i.e., the square of the NA ratio, $(0.8 / 1.2)^{2} \approx 0.44$. Combining these two considerations, we estimate $R_{\text {theo }}$ to a value of 0.22 to 0.25 .

Alternatively, we end up in the same range of values if we drop the dependence on the NA altogether and instead introduce an additional GRIN element as may be deduced from the sketch on the specification sheet. If this gap is filled with air and light rays pass this gap in a collimated fashion, we get $R_{\text {theo,alt }} \approx 0.26$ to 0.30 for a single on-axis ray and $R_{\text {theo,alt }} \approx 0.25$ to 0.30 for a full angular fan of 75 rays.

\subsection{Transmission Measurement}

To check the theoretical estimate for the transmissibility of the GRIN system mentioned above, the power of the stationary, non-scanning Ti:Sa laser beam has been measured with a laser power meter (841-PE, Spectra-Physics-Newport, Irvine, CA) both before entering and after exiting the GRIN system. These measurements yield values of 0.76 to 0.79 for the transmissibility of the GRIN system. Applying the same reasoning with respect to effective signal collection capability as above, we get a next estimate for the relative SHG signal level, $R_{\text {transmission }}$ $\approx 0.19$ to 0.22 .

\subsection{SHG Measurements}

Panels C and D of Figure 1 show examples of backscattered SHG imaging of muscle tissue recorded with the standard laser scanned two photon microscope and the GRIN lens based extended LSM set up, respectively. The typical striation pattern, the fundamental feature in microscopic SHG images from muscle tissue samples, can be clearly seen in both examples, although the absolute SHG signal level is significantly smaller for the GRIN system. In addition, the limited object field is clearly visible. Similarly, panels E and F show examples of SHG imaging of collagen in skin tissue.

Each SHG image was sampled with 512 pixels by 512 pixels in size. Background signal and background standard deviation were measured in gray scale units in an image region of interest outside the sample using MAT- 
LAB software (The Mathworks, Natick MA). Signal strength was quantified in same gray scale units from a region of interest in the center of the object field. Subsequently, the mean background value was subtracted from the signal gray scale values. This net SHG signal was divided by the square of the laser power to correct for varieties in the incident laser power and corrected for differences of the PMT amplification factor at the given voltage, see "Methods".

It is expected that the back-scattered SHG signal in muscle depends on the thickness of the sample [25]. To avoid this dependence and maximize the back-scattered signal, we have only included thick muscle samples with a diameter of more than $850 \mu \mathrm{m}(n=6$ measurements with the standard microscope, $n=13$ with the GRIN system). We then have calculated the signal ratio of the two mean values (GRIN vs. objective) and obtained a relative SHG signal level of $R_{\text {muscle }} \approx 0.30 \pm 0.08$.

Accordingly, we have analyzed the SHG images from thin skin tissue $(n=5$ measurements with the standard microscope, $n=5$ with the GRIN system) and get $R_{\text {skin }} \approx$ $0.31 \pm 0.06$ for the relative signal level. Figure 2 summarizes the theoretically and experimentally estimated relative signal levels mentioned in this study.

\section{Discussion}

The ratio of the net SHG signals obtained with either a standard two photon LSM or an extended GRIN-lens based LSM has been theoretically estimated and experimentally characterized. The GRIN lens system used in this study is a complete off-the-shelf system specifically designed for two-photon microscopy. Table 1 summarizes its advantages and disadvantages.

For our study, the focusing optics is the main physical difference between both systems, especially with respect to the numerical aperture where the nominal values differ substantially: 0.4 for the $10 \mathrm{x}$ lens, $0.18 / 0.8$ for the GRIN relay and 1.2 for the $63 \mathrm{x}$ lens. Yet, as has been mentioned before, we are underfilling the back aperture of both objective lenses which are approximately $14 \mathrm{~mm}$ and $8.5 \mathrm{~mm}$ in diameter for the $10 \mathrm{x}$ and $63 \mathrm{x}$ lens, respectively: With a beam diameter of approximately 5.5 $\mathrm{mm}$, only $2 / 3$ of the $63 \mathrm{x}$ lens' back aperture and only $40 \%$ of the $10 \mathrm{x}$ lens' back aperture are used, practically smoothing out any substantial differences between the excitation NA of both systems.

Yet, the difference in object-side NA will have an impact on the collection efficiency of the systems. Using a simple $\mathrm{NA}^{2}$ dependence, the standard microscope system should collect approximately twice as much photons as the GRIN system, which will suffer further signal loss due to the increased number of optical interfaces.

We have therefore set up a simple two component model for the GRIN lens to estimate the effect of these reflection losses and end up with a transmissibility that is in acceptable agreement with the experimental value (see "Transmission measurement" above). Furthermore, incorporating the $\mathrm{NA}^{2}$ dependence, we obtain a relative signal level $R_{\text {theo }}$ that fits well to the experimental results of $R_{\text {muscle }}$ and $R_{\text {skin }}$ :

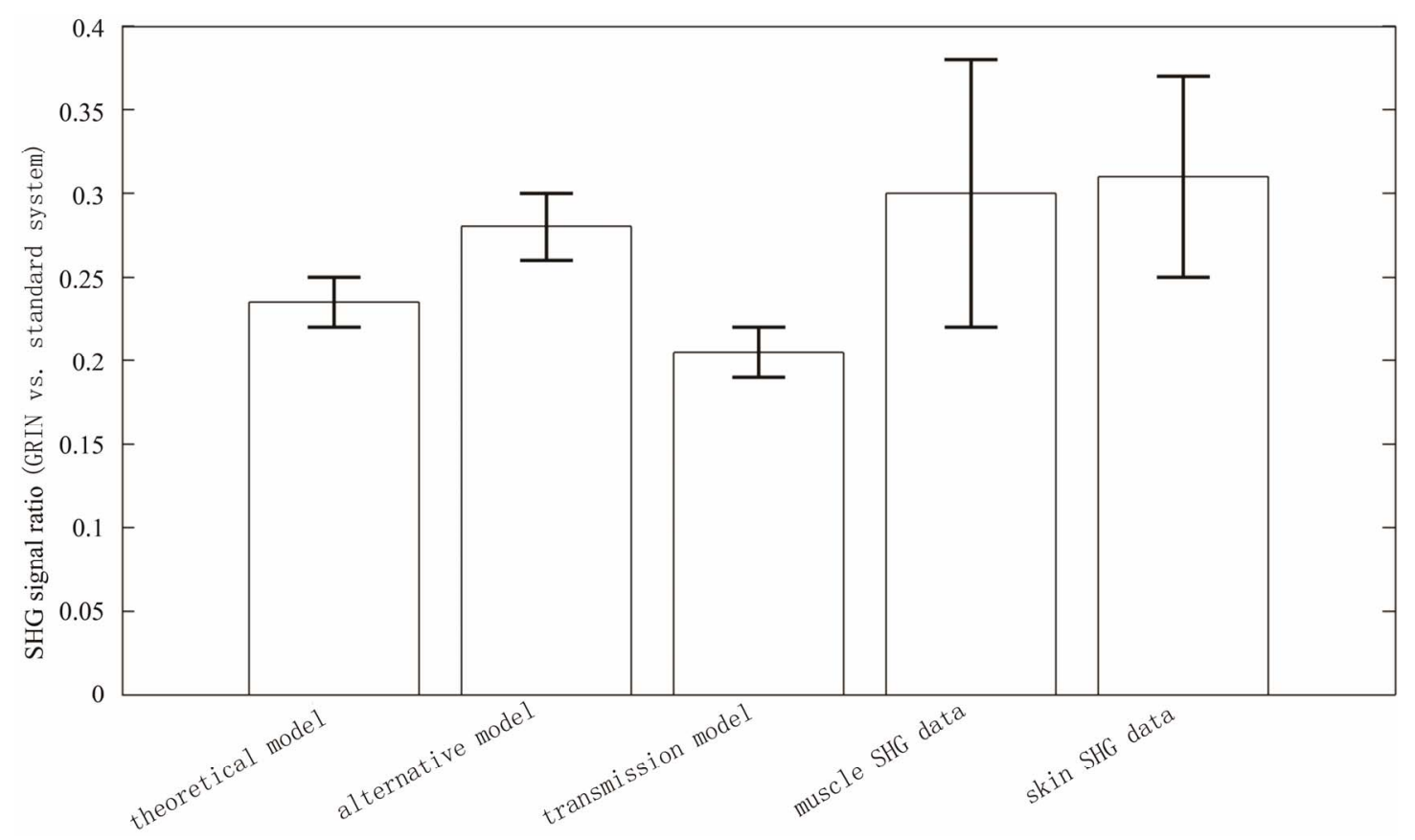

Figure 2. SHG signal ratio between the GRIN lens based LSM and the standard two photon microscope, based on theoretical estimates and experimental data, see text. 
Table 1. Pros and cons of the GRIN lens system used in the present study (GRIN-GT-MO-080-018-810, Grintech GmbH, Jena, Germany).

\begin{tabular}{|c|c|}
\hline \multirow{5}{*}{ PRO } & $\begin{array}{l}\text { - Commercially available off-the-shelf system, } \\
\text { optical parts aligned in metal rod case } \\
\text { Optimized for two photon excitation between } 800 \\
\text { nm and } 900 \mathrm{~nm} \text {, compatible with many standard } \\
\text { fluorescent dyes }\end{array}$ \\
\hline & $\begin{array}{l}\text { - Small pulse chirping effect due to short length } \\
(7.5 \mathrm{~mm})\end{array}$ \\
\hline & $\begin{array}{l}\text { - } 10 \mu \mathrm{m} \text { change in working distance only between } \\
-1000 \mathrm{~nm} \text { and } 700 \mathrm{~nm}\end{array}$ \\
\hline & $\begin{array}{l}\text { - Small optical aberrations, specified to } \\
\text { diffraction limited performance in the center of } \\
\text { the field }\end{array}$ \\
\hline & $\begin{array}{l}\text { - Performance on edge of field can be improved } \\
\text { with adaptive optics [4] }\end{array}$ \\
\hline $\mathrm{CON}$ & - Short system with limited penetration depth \\
\hline
\end{tabular}

The mean value of $R_{\text {theo }}=0.235 \pm 0.015$ is only $\approx 15 \%$ larger than the mean of $R_{\text {transmission }}=0.205 \pm 0.015$ and it is only 0.8 and 1.25 standard deviations apart from the mean values of $R_{\text {muscle }}$ and $R_{\text {skin }}$, respectively.

This is also why we are cautious with an alternative GRIN lens model that replaces the $\mathrm{NA}^{2}$ dependence with the introduction of additional internal optical interfaces although the resulting value of $R_{\text {theo,alt }}$ looks promising at first sight.

GRIN lens systems usually show significantly larger chromatic aberrations than classical microscope objective lenses. With the GRIN system at hand, which is optimized for biomedical multi photon imaging, the working distance is specified to shorten by $10 \mu \mathrm{m}$ for a wavelength shift from $1000 \mathrm{~nm}$ to $700 \mathrm{~nm}$, yet the effect should be smaller in the visible range (Grintech, personal communication). Overall, this defocus effect will result in a larger footprint of the SHG light in the intermediate coupling plane between the 10x and the GRIN lens, and it could lead to clipping effects in subsequent apertures. However, it seems that this effect is not hurting us substantially here.

An additional draw back in the use of GRIN lenses is the limited size of the object field which can be clearly seen in panels D and F of Figure 1. The signal decrease on the rim of the lens can be significantly reduced with adaptive optics [4]. We did not have such a system at our disposal, but our results should be generalizable to these cases as well.

In addition to these geometric optical properties, multi photon signal generation is also affected by the width of the laser pulses. For GRIN lenses, a group delay dispersion in the order of $10,000 \mathrm{fs}^{2}$ has been reported [31], so that femto-second pulses will see significant chirping when they pass through the lens. This chirping effect is however strongly reduced for a laser system as ours that outputs pulses 2 to $3 \mathrm{ps}$ in length, so that we think we can neglect any pulse stretching effects, or differences in pulse stretching, in the two lens systems.

For the experimental part of our study, we have used samples of high biomedical interest and recorded the intrinsic non-bleaching SHG signals from myosin and collagen in muscle and skin, respectively. Apart from these advantages, we made use of the important characteristic angular pattern seen in SHG emission: SHG from myosin is dominantly emitted in forward direction, whereas collagen shows emission to both hemispheres around the focal spot.

We have therefore used thick samples of "optically cleared" muscle to establish a less favoring experimental setting where the signal collection is complicated by the fact that any SHG photon reaching the detector must first be scattered back by the sample tissue with low light scattering efficiency.

Accordingly, SHG from thin skin samples will show no backscattering from photons emitted in forward direction, and the detector will only see SHG photons that are directly emitted in backward direction. This more favoring experimental setting is much more similar to fluorescence where signal is emitted isotropically.

It is very satisfactory that both samples yield very similar values for the relative level of SHG signal. Although the conditions for a Student's t-test are not completely met, we compare $R_{\text {muscle }}=0.30 \pm 0.08$ to $R_{\text {skin }}=$ $0.31 \pm 0.06$. If we conservatively assume that these intervals are generated by $n_{1}=19$ and $n_{2}=10$ independent measurements, we get a p-value of $\approx 0.71$. Likewise, to find a difference between the mean values for muscle and skin samples at the $\alpha=0.05 / \beta=0.1$ level, would need at least $n_{1}=n_{2}=1050$ measurements.

Thus, the relative level of SHG signal seems to be dominated by two factors only: the transmissibility of the additional GRIN relay and the difference in object-side NA, given, however, that the chromatic aberrations are small enough to neglect any clipping effects in the optical path.

We therefore think that the ratio values which we have obtained for the relative signal level in GRIN versus standard microscope systems can be generalized to other laser scanning modalities like confocal or multi photon fluorescence, SFG, THG or CARS, giving researchers in these fields some ground that GRIN technology could be a suitable solution if the original signal to noise ratio in standard LSM epi-detection is approximately four or higher. We furthermore conclude that GRIN-based laser scanning systems can make use of the optical and optoelectronic filters and detectors that are already well-established in standard laser scanning microscopes. 


\section{Acknowledgements}

The authors wish to thank Cornelia Weber for essential sample preparation work. This study was supported in part from the German Ministry for Education and Research (13N7871 to R.H.A.F.) and Landesforschungsschwerpunkt Baden-Württemberg (to R.H.A.F.). S. Schürmann was holding a scholarship (Landesgraduiertenförderung) from the state of Baden-Württemberg, Germany.

\section{REFERENCES}

[1] K. König, A. Ehlers, I. Riemann, S. Schenkl, R. Bückle and M. Kaatz, "Clinical Two-Photon Microendoscopy," Microscopy Research and Technique, Vol. 70, No. 5, 2007, pp. 398-402. doi:10.1002/jemt.20445

[2] P. Kim, M. Puoris'haag, D. Côté, C. P. Lin and S. H. Yun, "In Vivo Confocal and Multiphoton Microendoscopy," Journal of Biomedical Optics, Vol. 13, No. 1, 2008, Article ID: 010501. doi:10.1117/1.2839043

[3] Y. Wu, J. Xi, M. J. Cobb and X. Li, "Scanning FiberOptic Nonlinear Endomicroscopy with Miniature Aspherical Compound Lens and Multimode Fiber Collector," Optics Letters, Vol. 34, No. 7, 2009, pp. 953-955. doi:10.1364/OL.34.000953

[4] C. Wang and N. Ji, "Pupil-Segmentation-Based Adaptive Optical Correction of a High-Numerical-Aperture Gradient Refractive Index Lens for Two-Photon Fluorescence Endoscopy," Optics Letters, Vol. 37, No. 11, 2012, pp. 2001-2003. doi:10.1364/OL.37.002001

[5] D. M. Huland, C. M. Brown, S. S. Howard, D. G. Ouzounov, I. Pavlova, K. Wang, D. R. Rivera, W. W. Webb and C. Xu, "In Vivo Imaging of Unstained Tissues Using Long Gradient Index Lens Multiphoton Endoscopic Systems," Biomedical Optics Express, Vol. 3, No. 5, 2012, pp. 1077-1085. doi:10.1364/BOE.3.001077

[6] C. M. Brown, D. R. Rivera, I. Pavlova, D. G. Ouzounov, W. O. Williams, S. Mohanan, W. W. Webb and C. Xu, "In Vivo Imaging of Unstained Tissues Using a Compact and Flexible Multiphoton Microendoscope," Journal of Biomedical Optics, Vol. 17, No. 4, 2012, Article ID: 040505. doi:10.1117/1.JBO.17.4.040505

[7] M. E. Llewellyn, R. P. J. Barretto, S. L. Delp and M. J. Schnitzer, "Minimally Invasive High-Speed Imaging of Sarcomere Contractile Dynamics in Mice and Humans," Nature, Vol. 454, No. 7205, 2008, pp. 784-788. doi:10.1038/nature07104

[8] S. V. Plotnikov, A. M. Kenny, S. J. Walsh, B. Zubrowski, C. Joseph, V. L. Scranton, G. A. Kuchel, D. Dauser, M. Xu, C. C. Pilbeam, D. J. Adams, R. P. Dougherty, P. J. Campagnola and W. A. Mohler, "Measurement of Muscle Disease by Quantitative Second-Harmonic Generation Imaging," Journal of Biomedical Optics, Vol. 13, No. 4, 2008, Article ID: 044018. doi:10.1117/1.2967536

[9] O. Friedrich, M. Both, C. Weber, S. Schürmann, M. D. H. Teichmann, F. von Wegner, R. H. A. Fink, M. Vogel, J. S. Chamberlain and C. Garbe, "Microarchitecture Is Se- verely Compromised but Motor Protein Function Is Preserved in Dystrophic Mdx Skeletal Muscle," Biophysical Journal, Vol. 98, No. 4, 2010, pp. 606-616. doi:10.1016/j.bpj.2009.11.005

[10] W. Denk, J. H. Strickler and W. W. Webb, "Two-Photon Laser Scanning Fluorescence Microscopy," Science, Vol. 248, No. 4951, 1990, pp. 73-76. doi:10.1126/science. 2321027

[11] A. Zoumi, A. Yeh and B. J. Tromberg, "Imaging Cells and Extracellular Matrix in Vivo by Using Second-Harmonic Generation and Two-Photon Excited Fluorescence," Proceedings of the National Academy of Sciences of the United States of America, Vol. 99, No. 17, 2002, pp. 11014-11019. doi:10.1073/pnas.172368799

[12] W. R. Zipfel, R. M. Williams, R. Christie, A. Y. Nikitin, B. T. Hyman and W. W. Webb, "Live Tissue Intrinsic Emission Microscopy Using Multiphoton-Excited Native Fluorescence and Second Harmonic Generation," Proceedings of the National Academy of Sciences of the United States of America, Vol. 100, No. 12, 2003, pp. 7075-7080. doi:10.1073/pnas.0832308100

[13] S.-H. Chia, C.-H. Yu, C.-H. Lin, N.-C. Cheng, T.-M. Liu, M.-C. Chan, I.-H. Chen and C.-K. Sun, "Miniaturized VideoRate Epi-Third-Harmonic-Generation Fiber-Microscope,' Optics Express, Vol. 18, No. 16, 2010, pp. 17382-17391. doi:10.1364/OE.18.017382

[14] G. Liu, K. Kieu, F. W. Wise and Z. Chen, "Multiphoton Microscopy System with a Compact Fiber-Based Femtosecond-Pulse Laser and Handheld Probe," Journal of Biophotonics, Vol. 4, No. 1-2, 2010, pp. 34-39. doi:10.1002/jbio.201000049

[15] M. Rehberg, F. Krombach, U. Pohl and S. Dietzel, "Signal Improvement in Multiphoton Microscopy by Reflection with Simple Mirrors near the Sample," Journal of Biomedical Optics, Vol. 15, No. 2, 2010, Article ID: 026017. doi:10.1117/1.3374337

[16] S. Roth and I. Freund, "Second Harmonic Generation in Collagen," Journal of Chemical Physics, Vol. 70, No. 4, 1979, pp. 1637-1643. doi:10.1063/1.437677

[17] P. J. Campagnola, A. C. Millard, M. Terasaki, P. E. Hoppe, C. J. Malone and W. A. Mohler, "Three-Dimensional High-Resolution Second-Harmonic Generation Imaging of Endogenous Structural Proteins in Biological Tissues," Biophysical Journal, Vol. 82, No. 1, 2002, pp. 493508. doi:10.1016/S0006-3495(02)75414-3

[18] M. Both, M. Vogel, O. Friedrich, F. von Wegner, T. Künsting, R. H. A. Fink and D. Uttenweiler, "Second Harmonic Imaging of Intrinsic Signals in Muscle Fibers in Situ," Journal of Biomedical Optics, Vol. 9, No. 5, 2004, pp. 882-892. doi:10.1117/1.1783354

[19] S. V. Plotnikov, A. C. Millard, P. J. Campagnola and W. A. Mohler, "Characterization of the Myosin-Based Source for Second-Harmonic Generation from Muscle Sarcomeres,' Biophysical Journal, Vol. 90, No. 2, 2006, pp. 693-703. doi:10.1529/biophysj.105.071555

[20] T. Boulesteix, E. Beaurepaire, M.-P. Sauviat and M.-C. Schanne-Klein, "Second-Harmonic Microscopy of Unstained Living Cardiac Myocytes: Measurements of Sarcomere Length with 20-nm Accuracy," Optics Letters, 
Vol. 29, No. 17, 2004, pp. 2031-2033. doi:10.1364/OL.29.002031

[21] G. Recher, D. Rouède, C. Tascon, L.-A. D'Amico and F. Tiaho, "Double Band Sarcomeric SHG Pattern Induced by Adult Skeletal Muscles Alteration during Myofibrils Preparation," Journal of Microscopy, Vol. 241, No. 2, 2011, 207-211. doi:10.1111/j.1365-2818.2010.03425.x

[22] S. Schürmann, F. V. Wegner, R. H. A. Fink, O. Friedrich and M. Vogel, "Second Harmonic Generation Microscopy Probes Different States of Motor Protein Interaction in Myofibrils," Biophysical Journal, Vol. 99, No. 6, 2010, pp. 1842-1851. doi:10.1016/j.bpj.2010.07.005

[23] L. Moreaux, O. Sandre, M. Blanchard-Desce and J. Mertz, "Membrane Imaging by Simultaneous Second-Harmonic Generation and Two-Photon Microscopy," Optics Letters, Vol. 25, No. 5, 2000, pp. 320-322. doi:10.1364/OL. 25.000320

[24] L. Moreaux, O. Sandre, S. Charpak, M. Blanchard-Desce and J. Mertz, "Coherent Scattering in Multi-Harmonic Light Microscopy,” Biophysical Journal, Vol. 80, No. 3, 2001, pp. 1568-1574. doi:10.1016/S0006-3495(01)76129-2

[25] F. Légaré, C. Pfeffer and B. R. Olsen, "The Role of Backscattering in SHG Tissue Imaging," Biophysical Journal, Vol. 93, No. 4, 2007, pp. 1312-1320. doi:10.1529/biophysj.106.100586
[26] R. LaComb, O. Nadiarnykh, S. Carey and P. J. Campagnola, "Quantitative Second Harmonic Generation Imaging and Modeling of the Optical Clearing Mechanism in Striated Muscle and Tendon," Journal of Biomedical Optics, Vol. 13, No. 2, 2008, Article ID: 021109. doi:10.1117/1.2907207

[27] S. Plotnikov, V. Juneja, A. B. Isaacson, W. A. Mohler and P. J. Campagnola, "Optical Clearing for Improved Contrast in Second Harmonic Generation Imaging of Skeletal Muscle," Biophysical Journal, Vol. 90, No. 1, 2006, pp. 328-339. doi:10.1529/biophysj.105.066944

[28] O. Nadiarnykh and P. J. Campagnola, "Retention of Polarization Signatures in SHG Microscopy of Scattering Tissues through Optical Clearing," Optics Express, Vol. 17, No. 7, 2009, pp. 5794-5806. doi:10.1364/OE.17.005794

[29] F. von Wegner, S. Schurmann, R. Fink, M. Vogel and O. Friedrich, "Motor Protein Function in Skeletal MuscleA Multiple Scale Approach to Contractility," IEEE Transactions on Medical Imaging, Vol. 28, No. 10, 2009, pp. 1632-1642. doi:10.1109/TMI.2009.2026171

[30] "Refractive Index Database," http://refractiveindex.info

[31] A. Ehlers, "Klinische Anwendungen der Multiphotonen-Tomographie Humaner Haut," Universitäts-und Landesbibliothek, Saarbrücken, 2008. 